\title{
Research on Control and Switch of Multi Operate Modes in a Micro Weak County Power Grid
}

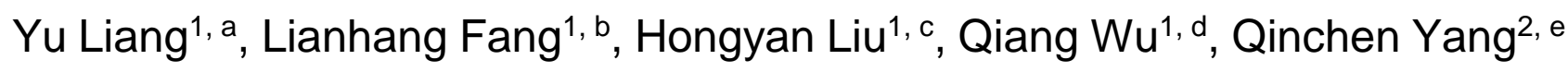 \\ ${ }^{1}$ Hainan Electric Power Research Institute, Haikou 570125, Hainan Province, China; \\ ${ }^{2}$ School of electrical engineering, Southeast University, Nanjing 210096, Jiangsu Province, China. \\ aliangyu66@foxmail.com, b71859943@qq.com, c15194868632@163.com, dqwu2006@163.com, \\ e409022938@qq.com
}

\begin{abstract}
When the grid structure area of county power grid is weak, its fragility is exposed in the face of major natural disasters (such as typhoon), which would result in serious consequences such as power loss in the whole city. If the traditional small hydropower resources of regional county power grids are abundant, by carrying out the regional multi-operation mode control and constructing the dispatching assistant decision-making system, the regional power grid can also continue to supply important load in the face of major natural disasters. In this paper, in terms of a county-level power grid characteristics, the operation and control technology of the fragile county-level rural power grids under the risk model is studied to construct the demonstration project, improve the ability of resisting the risk of regional power grid and ensure the important load of county power supply. The effectiveness of the decision system is verified by simulation analysis in the actual power grid.
\end{abstract}

Keywords: Regional Power Network; Normal Operation Mode; Risk Operation Mode; Operation Control.

\section{Introduction}

For the research of micro grid, the main current focus on system stability analysis, power quality control, energy optimization management and normal / island smooth conversion and other aspects. Most of the existing micro-power capacity optimization methods are aimed at optimizing the economic cost, reference [1-3] proposes a method of optimal allocation of micro-power source with optimal energy recovery cost, can be in the micro-grid life cycle to maximize the use of renewable energy output. The dynamic models of micro-power supply, such as micro-gas turbine, wind power generation, fuel cell and photovoltaic cell, are established in reference [4-5], and analyzes the characteristics of various distributed power supplies. Reference [6-8] introduces the challenges of large-scale distributed power access micro grids and users' demand for high-level security and power quality. However, there are few researches on micro-grid operation control for distributed rotating electrical machines, especially in the face of risks, regional power grid control mode of operation at domestic and foreign research literature is relatively small.

As a county-level regional power grid in Hainan is rich in traditional small hydropower resources, no wind, light, storage and other new energy sources, Therefore, the power supply of the regional power grid is in synchronous rotation state; and there is no micro-grid concept in a large number of power electronic devices currently. therefore, this paper intends to study "regional grid operation control technology research and engineering application under risk mode" in a certain county area of Hainan province, the development of multi-mode power grid operation controller, to improve the regional power grid to combat extreme natural disasters from the technical level, to ensure that the county under the natural disaster to continue to provide electricity. In this paper, multi-mode operation control of micro-grid based on hydropower access is carried out to improve the reliability of regional micro-grid power supply.

\section{Regionalization of micro grids}

After a large number of distributed power accessed to the grid, obtaining benefits and affecting the safe operation of traditional power grid at the same time, such as changing the system current, 
resulting in voltage disturbance under system breakdown, power angle instability and so on. The micro grid is flexible in its structure and operation mode through its distributed power supply and diversity load, can be through effective micro-grid management logic, distribution network and micro-grid coordination and control system, to weak or even eliminate the risk of distributed power accessed to power grid operation, to give full play to the technical advantages of flexible and efficient operation of power grid under micro-grid connection. When the regional grid is actively or passively disconnected from the large grid, it is possible to focus on maintaining the internal power balance of the micro-grid and the power supply of the important load, the division of micro-grids into regional power grids firstly [9].

At present, there are many methods of regional division of microgrids, mainly based on the electrical distance of the island division, based on the voltage stability index division, based on the voltage stability margin of the island division and power balance based on the division strategy [10]. This paper mainly consider the actual distribution network power supply structure and load distribution, a method of micro grid division based on power balance is adopted.

Firstly introduce the concept of power balance, the power balance is represented by a power balance coefficient:

$$
\lambda=\Delta P / S_{D G}
$$

$\Delta P$ is connected to the lower unit micro-network feeder trend, which corresponds to the power unbalance amount of the lower layer micro grid; represents the total DG capacity corresponding to the lower electrical island; Assuming that the positive direction of the current flows from the public grid to the micro grid, the power balance degree is defined as:

$\left\{\begin{array}{l}\lambda>0 \Rightarrow S_{L D}>S_{D G} \\ \lambda=0 \Rightarrow S_{L D}=S_{D G} \\ \lambda<0 \Rightarrow S_{L D}<S_{D G}\end{array}\right.$

$S_{L D}$ represents the capacity of the load corresponding to the lower electrical island, can be based on power balance to be taken into account whether the regional grid for micro-network division.

\section{Micro-grid multi-mode operation}

In order to improve the ability of regional power grids to resist extreme natural disasters and ensure the continuous power supply of important loads, the operation modes of regional power grids are divided into normal operation mode, risk operation mode, isolated network operation mode and return to normal operation mode. For different modes, the corresponding micro-network control strategy and stability control strategy are studied.

The regional power grid dispatching aided decision system is developed, which is composed of intelligent dispatching auxiliary support system, multi-mode micro-grid controller, small hydropower automatic optimization control device and so on, which can realize the economic dispatch of small hydropower station in normal mode, improve power generation efficiency; achieve the effective operation of the risk control of regional power grids large power grid transmission power transmission line; ensure the critical load normal power supply in the isolated network; achieve rapid grid operation; and provide protection to the power grid economy and safe operation.

\subsection{Normal operation mode}

Normal operation mode is based on the normal operation mode of the power grid and normal climatic conditions. Under the normal operation mode, the micro-grid controller collects the situation of the small hydropower network and the loading situation, and forwards the information to the dispatching assistant decision system.

On the basis of fully analyzing and investigating the power grid, this paper analyzes the output characteristics of regional small hydropower by combining the theory with the actual research method. There are different daily, seasonal and real-time regulation characteristics of Small Hydropower Groups. To establish a stable power supply in the complementary micro-grid, it should schedule a planning and real-time scheduling for different time periods and different regions micro-grid power 
supply, combined with the prediction characteristics, And realize the continuity and smoothness of the overall power generation plan of regional power grids. The modeling and analysis with uncertain thinking and methods, the establishment of multi-objective, and multi-constraint configuration model are keys to optimal configuration. The establishment of hydropower station group power generation MAXMIN model which ensures the maximum output as the optimization criteria is as follows:

$$
\begin{aligned}
& f=\max \left\{\operatorname{minN}_{S j}\right\} \\
& \mathrm{N}_{S j}=\sum_{i=1}^{n} \mathrm{~N}_{i, j} ;(\mathrm{j}=1,2,3 \cdots, \mathrm{N})
\end{aligned}
$$

Where $\mathrm{i}$ is the $\mathrm{i}$-th power station, $\mathrm{j}$ is the $\mathrm{j}$-th time period, $\mathrm{n}$ is the number of participating hydropower stations, $\mathrm{N}$ is the output, and $\mathrm{f}$ is the total output of the hydropower station.

In this paper, based on the normal system model of small hydropower group established during the normal operation, the grid loss is minimized and the active power of the hydropower station is used as the control variable in a scheduling cycle.

$\min F=\Delta t * P \_$loss

Where, $\Delta \mathrm{t}$ is the sampling time, $\mathrm{P}$ loss is the network loss. The model also includes active power balance constraint, branch transmission capacity constraint, hydropower unit climbing rate constraint and output constraint, and voltage constraint, and so on.

\subsection{Risk operation mode}

Risk operation mode is to advance to the emergency mode before the advent of typhoon and other extreme weather to prevent power outage in the county caused by wind and rain. Under the risk operation mode, the micro-grid controller monitors the operation status of the regional micro-grid and the large-grid tie-line, the load in the substation, the condition of the small hydropower network, the operation isolation criterion and the relevant stability control strategy, puts the tripping outlet platen, control tie line transmission power, and prepare for a smooth transition to the isolated network operation mode.

The active entry to the risk mode is based on the meteorological warning information and the risk warning level before the coming of extreme weather. The dispatcher orders to put exit platen in function and sets the operation mode to "risk operation mode" in the scheduling auxiliary decision system. Passive entry to the risk operation mode is that the regional power grid disconnected part of the contact lines with the large power grid when suddenly encountered strong bad weather, when the regional grid goes into the risk operation mode. The specific logic diagram is shown in Fig.1.

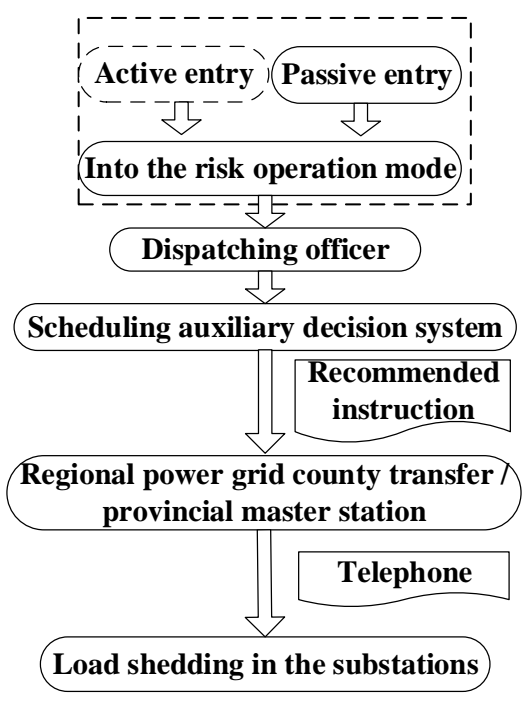

Fig. 1 Logic diagram of risk operation mode

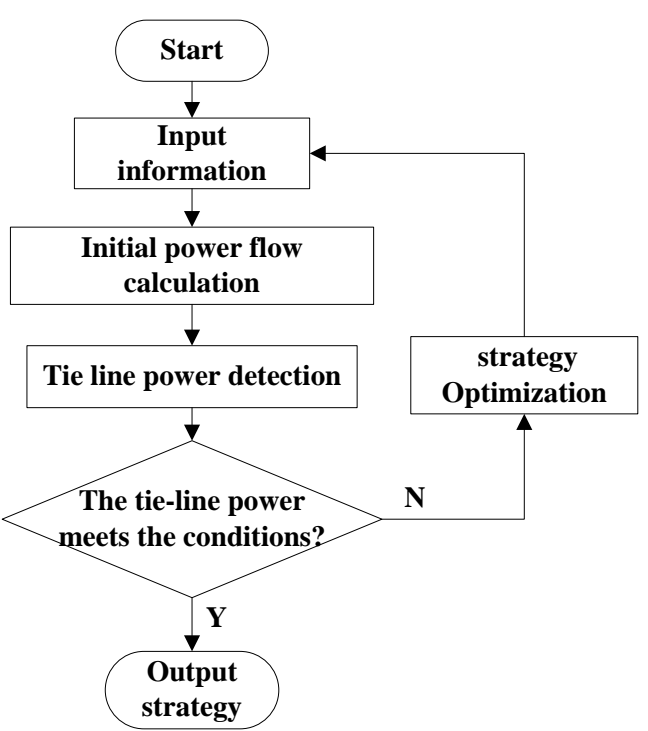

Fig. 2 Logic diagram of risk operation program
cision system when enter into the different risk op-eration mode by judging, control the forward tie line transmission power as small as possible according to the built-in optimization program when the specific action takes place, and calculate load 
shedding according to the load level. The theoretical power flow calculation is simulated in BPA and MATLAB, and can be calculated according to real-time hydropower generation output and load information. The concrete logic is shown in Fig.2.

\subsection{Isolated network operation mode}

Isolated network operation mode is a mode of operation after a trip of the connection line between the regional grid and the main network.

In the risk operation mode, the micro-grid con-troller monitors the operating status of the tie-line. When the isolation criterion is met, the regional grid is automatically broken down into multiple small mi-cro-grids. In this mode, small hydropower outputs and micro-network loads are coordinated, part of the load are cut to achieve the balance of source and load, and to ensure stable operation of micro-network and im-portant load non-stop.

When the detection shows the system runs in the isolated network operation mode, according to the scheduling support decision-making system, cut off the contact line quickly and the system goes into a number of isolated network state. In order to ensure sustained and stable power supply of important load in substation, when the hydropower output is insufficient to support the entire substation load, some loads are orderly re-moved according to the load level, making the stable operation of the small isolated network.

\subsection{Recovery operation mode}

The recovery operation mode is that after the ex-treme weather, the grid has resumed normal operation, and it needs a normal connection between the isolated network and the main network.

The reliability and stability of the isolated network operation is not as good as normal operation. When the fault of the main network contact line is eliminated, the reconnection of the county network to the main net-work should be arranged as soon as possible. As the frequency and voltage of the two systems are not con-sistent, in order to avoid the impact of the normal process on the isolated network and the main network, the normal conditions of the isolated network should be analyzed.

Firstly, the voltage difference between the isolated network and the main network is as small as possible, and the voltage difference will lead to the fluctuation of the normal frequency and power, resulting in the in-stability of the system. Secondly, the frequency of the isolated network should be slightly lower than the main network frequency, because the power flows from the high frequency of the main network to the low fre-quency of isolated network, reducing the operational pressure of small hydroelectric generating unit. (Due to the fact that both will eventually run at the same fre-quency, the power gap of the one with lower frequency will be filled by the one with higher frequency). Finally, the voltage of the main network must be ahead of the isolated network voltage at normal time, because the power flows from the voltage leading node to the one with phase lag voltage, reducing the oscillation of small hydroelectric generating unit at normal state.

\section{Study on Switching Strategy between Multi-operation Modes of Micro-grid}

\subsection{Switching from normal to risk operation mode}

When the normal operation of the regional grid switches to the risk mode, the contact line is not completely disconnected, and the risk mode operation is also a special state of the normal mode operation. The operation of the regional power grid in the risk mode is supported by only one tie-line, and is more fragile compared with normal mode operation. At this time, transmission power is controlled and the specific program is given. When the regional power grid switch to the risk operation mode actively or passively. A single tie-line can support the regional power grid load in a short time. The decision support system will provide a recommended strategy for scheduling to control tie-line power so that the regional grid does not over-reliance on large power grids to prepare for the smooth entry into the isolated network.

\subsection{Switching from risk to island mode}

When the regional power grid runs in risk mode, the power of the tie-line is controlled. If tie-line disconnects actively or passively at this time, the regional power network is solitary. In the isolated-grid model, the study object is an independent small-solitary network, and the outside part of 
the grid can be regarded as a static load. Since the transmission power of the tie-line has been controlled in a small range in the risk operation mode, the loss of this static load can be considered as a sudden loss of power due to the unit failure or sudden load interruption due to a line fault.

\section{Case Analysis of a County-level Regional Power Grid in Hainan Province}

The case analysis of this paper is based on a county-level regional power grid in Hainan Province and the grid structure of the county-level regional power grid is shown in Fig 6. Station WL is connected with large power grid, and line JL $(35 \mathrm{kV})$, line WL $(110 \mathrm{kV})$ and line WXL $(110 \mathrm{kV})$ is the tie line between county-level regional power grid and large power grid.

In this paper, once the contact line breaks down, the county will enter the isolated net operating status. As hydropower station F is connected with main network tie line in T-type connection and it can't be powered when the tie-line is disconnected. According to the assumption that run-of-river hydroelectric station stop to generate electricity when typhoon arrives, only hydropower station A and B work for the isolated network power supply. Because the unit type and the adjustment way of hydropower station A and B are different, when two hydropower stations provide the electric power support to the same substation simultaneously, it will cause the power mutually to grab and difficult to adjust. So the supply mode that a single hydropower station connects to a single substation power is used. The regional grid is divided into two areas: station LD+ hydropower station A (110kV) and station BY + hydropower station B $(35 \mathrm{kV})$. The solitary network is decomposed into two independent small solitary networks which is shown in Fig 4.

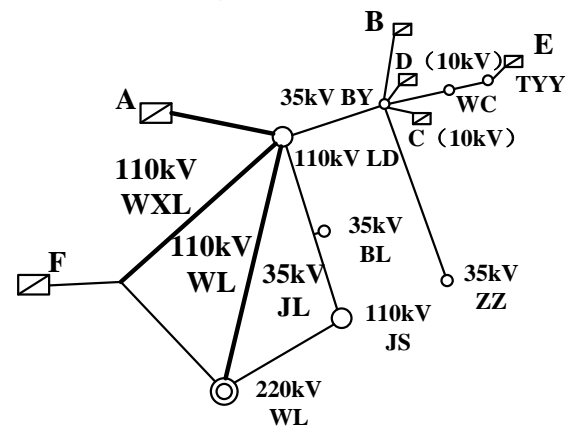

Fig. 3 County-level regional power grid structure

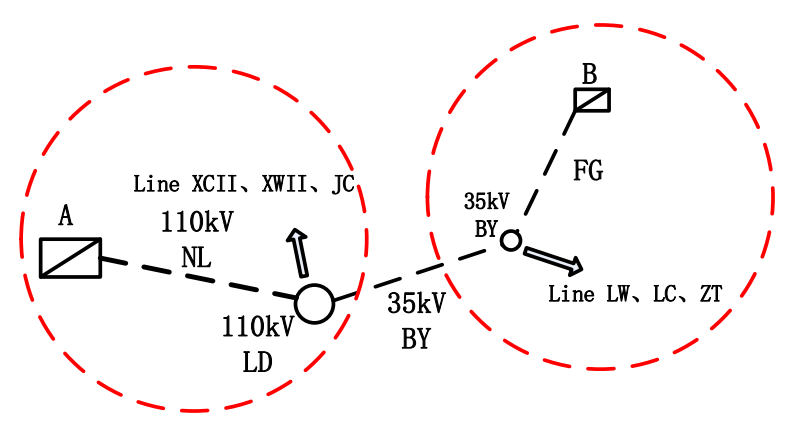

Fig. 4 The isolated network topology of the county regional power grid

In the normal operation, the minimum power loss of the county-level regional power grid is taken as the objective function, and the intelligent algorithm is used to optimize the analysis. The best power arrangements of water storage hydropower station is shown in Table1.

Table 1 The arrangement of the power output of the hydropower

\begin{tabular}{ccc}
\hline hydropower station A & hydropower station B & hydropower station F \\
\hline $8460 \mathrm{~kW}$ & $1840 \mathrm{~kW}$ & $4000 \mathrm{~kW}$ \\
\hline
\end{tabular}

In the risk operation mode, the transmission power of the tie-line is controlled. At time b, before the load is cut or outputted, the transmission power of line WXL is $8390 \mathrm{~kW}$ and the transmission power of line BY is $2930 \mathrm{~kW}$. After the load is cut or outputted, the transmission power of line WXL is $2600 \mathrm{~kW}$ and the transmission power of line BY is $6.2 \mathrm{~kW}$. The results show that the method reduce 
the tie-line transmission power effectively, hydropower output and load removal arrangements as shown in Table 2 and Table 3.

Table 2 Arrangement of hydropower output

\begin{tabular}{ccc}
\hline name & initial & control plan \\
\hline hydropower station E & $800 \mathrm{~kW}$ & unchanged \\
hydropower station D & $800 \mathrm{~kW}$ & unchanged \\
hydropower station C & $800 \mathrm{~kW}$ & unchanged \\
hydropower station B & $800 \mathrm{~kW}$ & unchanged \\
hydropower station A & $6000 \mathrm{~kW}$ & unchanged \\
\hline
\end{tabular}

If the tie-line is disconnected, the county-level regional grid enters an isolated network with the isolated network frequency shown in Figure 5 and Figure 6. It can be seen that the isolated network frequency can meet the requirements of users, and it can maintain the stable and effective verification of the auxiliary decision support system to ensure the stable operation of the county-level regional power grid.

Table 3 Load shedding schedule

\begin{tabular}{cccc}
\hline Substation & Name of 10kV line & Load Importance Level & Resection(1 for a reservation, 0 for removal) \\
\hline \multirow{5}{*}{ LD } & LD load1 & 1 & 1 \\
& LD load 2 & 1 & 1 \\
& LD load 3 & 1 & 1 \\
& LD load 4 & 2 & 0 \\
& LD load 5 & 2 & 0 \\
& LD load 6 & 2 & 0 \\
\hline \multirow{5}{*}{ BY load 7 } & 2 & 0 \\
& BY load 1 & 1 & 1 \\
& BY load 2 & 1 & 1 \\
& BY load 3 & 1 & 1 \\
& BY load 4 & 2 & 1 \\
& BY load 5 & 2 & 1 \\
& BY load 6 & 2 & 1 \\
& BY load 7 & 2 & 1 \\
\hline \multirow{3}{*}{ ZZ } & BY load 8 & 2 & 0 \\
& ZZ load 1 & 3 & 0 \\
& ZZ load 2 & 3 & 1 \\
\hline
\end{tabular}

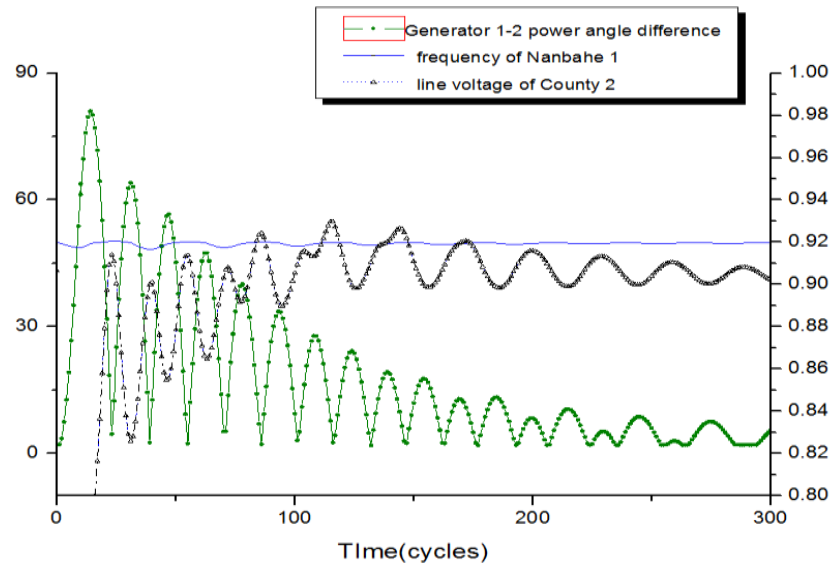

Fig. 5 The minimum bus frequency, voltage and maximum generator power angle difference of NO.1 isolated network 


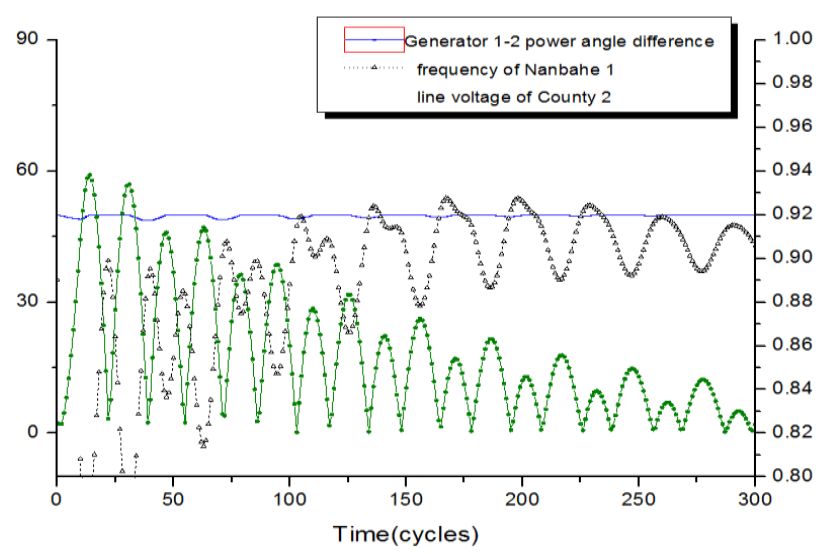

Fig. 6 The minimum bus frequency, voltage and maximum generator power angle difference of NO.2 isolated network

From the simulation results, it can be seen that the grid can maintain the stability of the micro-grid through the series of actions such as reducing the load when facing the risks. Thus it is ensured that the continuous power supply of important load.

\section{Summary}

In this paper, the multi-operation mode control and switching of regional micro-grid are studied. Based on the analysis and research of the specific regional power grids, the theory combined with the actual research method is used to study the regional power grid in the multi-operation mode analysis. When faced with natural disasters, it is enhanced that the ability of the regional power grid to resist risks, and it is ensured the continuous power supply to the county's critical loads. The feasibility of dispatching decision-making system is analyzed and simulated by a county - level regional power network in Hainan province.

\section{Acknowledgements}

Project supported by China Southern Power Grid science and technology project 'Researches on operation and control technologies and engineering application of Ledong grid under risk mode'.

\section{References}

[1] Dache Valentin, Caraivan Mitrut Corneliu, Sgârciu Valentin. Advanced Building Energy Management Systems, Optimize power consumption using IP Base Control Improved Power Grid Stability [J]. IFAC Proceedings Volumes, 2012,456.

[2] Germane Xavier Athanasius, Hemanshu R. Pota. Robust Decentralized Switching Power System Stabilisers for Interconnected Power Grids: Stability using Dwell Time [J]. IFAC Proceedings Volumes, 2008,412.

[3] Tristan G. Magallones, Jai Govind Singh, Watcharakorn Pinthurat. Power Flow and Small Signal Stability Analysis on the Interconnected Three Isolated Philippine Power Grid [J]. Perspectives in Science, 2016.

[4] Zeng Z, Zhao R X, Yang H. Micro-sources design of an intelligent building integrated with micro-grid [J]. Energy and Buildings, 2013, 57: 261-267.

[5] Liu Zhengyi, Tan Shuntao, Zeng Xiangjun. Distributed generation and its impact on power system analysis [J]. North China Electric Power, 2004(10): 18-20.

[6] Lasseter R. Dynamic models for micro-turbines and fuel cells[C]//Power Engineering Society Summer Meeting. Canada: Institute of Electrical and Electronics Engineers Inc., 2001: 761-766. 
[7] Naka S, Genji T, Fukuyama Y. Practical equipment models for fast distribution power flow considering interconnection of distributed generators [C] //IEEE Power Engineering Society Summer Meeting. Vancouver: Institute of Electrical and Electronics Engineers Inc., 2001: 1007-1012.

[8] Liu Zhengyi, Zeng Xiangjun, Tan Shuntao, et al. A novel scheme of stability control for distributed generation systems [C] //2004 International Conference on Power System Technology. Singapore: Institute of Electrical and Electronics Engineers Inc., 2004: 1528-1531.

[9] LIU Chang, YUAN Rongxiang1, LIU Bin, et al. Research on Microgrid Operation and Development [J]. Southern Power System Technology, 2010, 04(5):43-47.

[10] YANG Zhenyu, XU Sheng, XU Xingtao. Comparison and analysis of high voltage large capacity D STATCOM main circuit [J]. Electric Power Automation Equipment, 2011, 31(6):55-59. 